Military Technical College

Kobry El-Kobbah, Cairo, Egypt

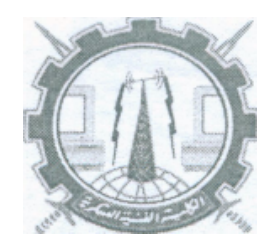

ICEENG $7^{\text {th }}$ International Conference

on Electrical Engineering

ICEENG 2010

\title{
Performance Analysis of Pseudo-Random Radars Identification Using Hidden Markov Model \\ By
}

Hossam Eldin Abou-Bakr Hassan*

\section{Abstract:}

Nowadays, radar signals are automatically adjusted by computer software to maximize radar performance. These signals are no longer stable and different pulse repetition interval (PRI) values may be assigned for each radar. Therefore, new, sophisticated (Electronic Surveillance), (Electronic Alert), and (Electronic Intelligence) signal processing algorithms are needed. This is the motivating factor behind the research on using HMM, for Radar System Identification (RSI). This paper, investigates the HMM recognition performance for identifying a pseudorandom PRI radar that randomly selects its PRI value from a number of fixed values. When pseudo-random sequences are considered, it becomes much more difficult to find an optimal HMM parameters that describe the dynamic behavior of the pseudorandom PRI radar. It will be shown that sub-optimal HMM parameters can still provide good recognition performance. Artificial pseudo-random PRI radar pulses are used to show that applying HMMs can provide adequate signal identification that is far superior to conventional cross-correlation techniques. The simulation results show that, it is necessary to retrain the HMM with an error-corrupted version of the original training sequence to improve the model's robustness.

\section{Keywords:}

Hidden Markov Model (HMM), Radar System Identification (RSI), Pulse Repetition Interval (PRI).

\section{Introduction:}

It is shown in $[1,2]$ that representing a radar system as a finite state machine is a convenient way to analyze its dynamic behavior for three main reasons. First, a radar system's output is controlled by events (manual or software driven) just like a finite

* Egyptian Armed Forces 
state machine. Second, the size of the state machines is not restricted. This means that any measurable characteristic of radar signal can be represented by one of these output symbols. Third, extensive analysis of finite state machine can be performed using HMMs [3-9]. It is shown in [1,2] that, the best number of states used for identifying a deterministic radar sequence, is directly related to the pulse train period. The number of observable symbols defines the number of discrete output alphabet. In RSI, the alphabet size is limited to two and is define the alphabet by $V=\{0,1\}$. A ' 0 ' represents a clock cycle where no pulse is present while a ' 1 ' represents a pulse of one clock period as shown in Figure 1 and Figure 2.

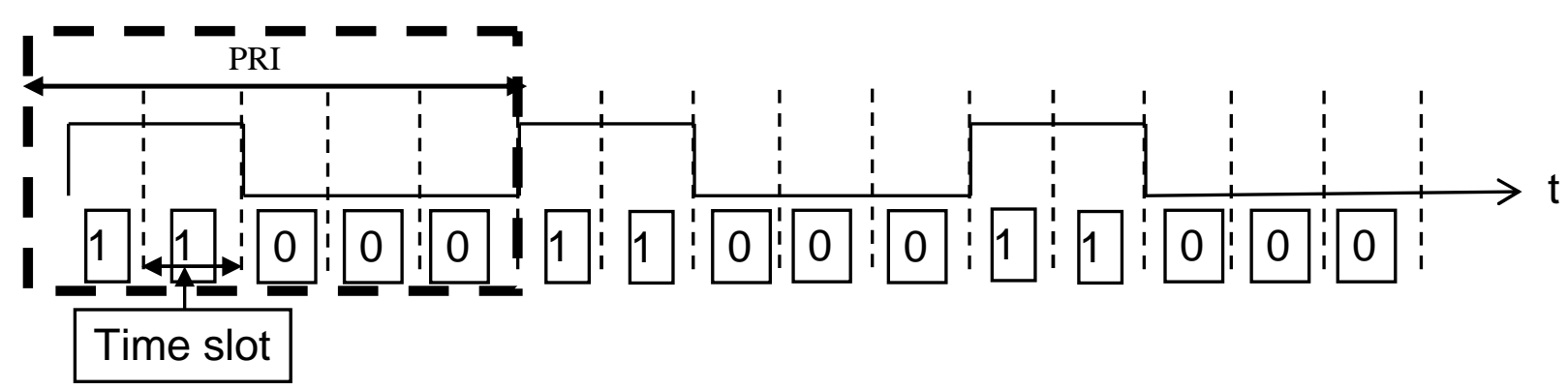

Figure (1): Observed deterministic PRI radar sequence, number of states $=5$

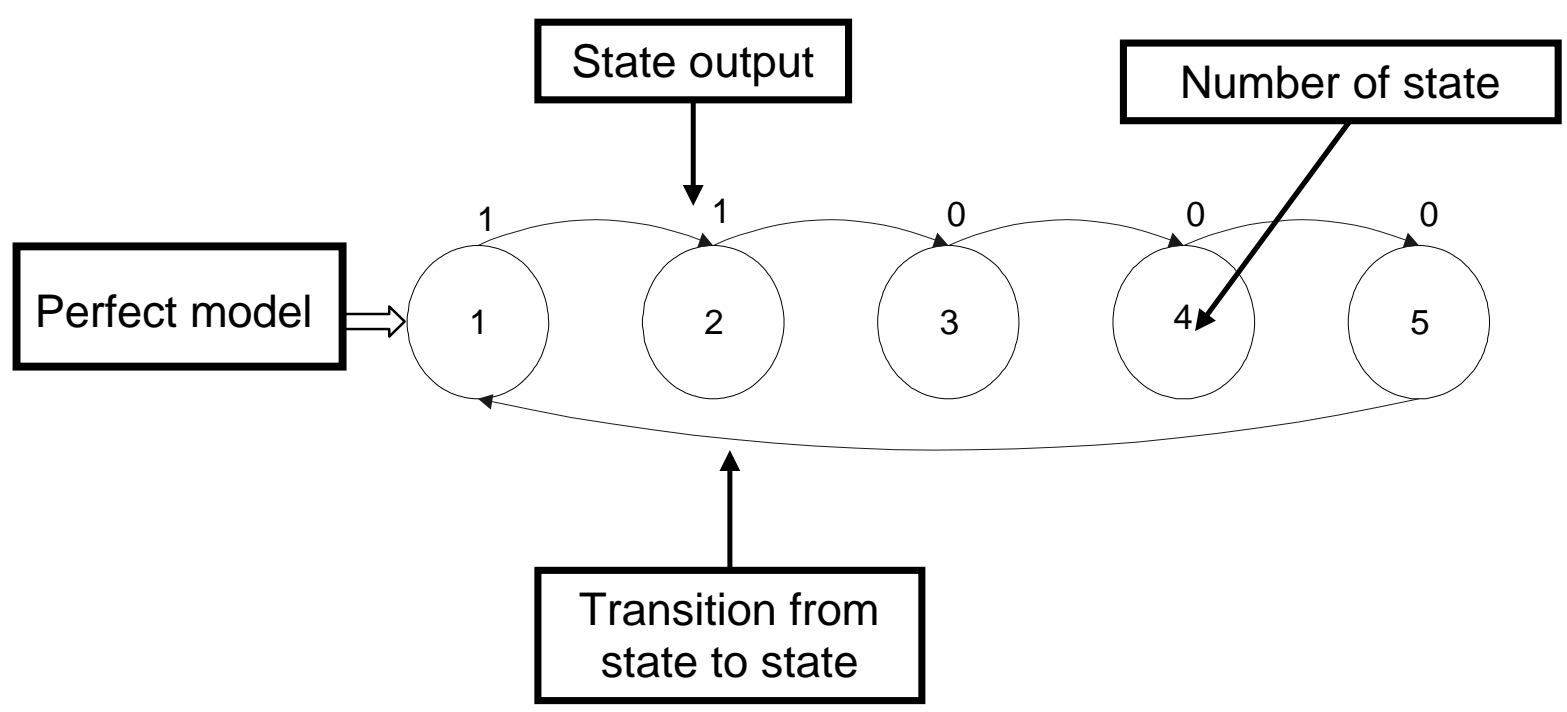

Figure (2): The dynamic behavior of the radar as a finite state machine

In [2], the training probability for a HMM is investigated as a function of the number of states chosen for the model. It was found that a perfect training probability can be realized as long as the number of states is equal to at least the number of symbols (transmitter clock cycles) per sequence period. The perfect model, however, must be slightly adjusted in order to accommodate for errors in an error-corrupted test sequence. The concept of retraining the perfect HMM to accommodate for observation errors, however, actually increases the false recognition rate because the competing models used have seen to benefit much more from this than does the correct model. Of course, the recognition performance depends on the error levels of both the retraining and observation sequences. Therefore, it is not correct to say 
universally that retraining degrades a library's recognition performance since many factors are involved. This is because an observation sequence will, on average, rarely exceed a rate of $10 \%$ dropped and 5\% spurious pulses.

Purely deterministic PRI radar sequences will, in general, rarely be produced from any modern radar [10] and, even if they were, the use of a HMM will offer little advantage over the conventional cross-correlation technique for RSI. They do, however, provide crucial knowledge about the behavior of a HMM under various circumstances $[1,2]$. This paper applies this knowledge to the more realistic case of having to design an optimal model for a pseudo-random PRI radar sequence. The theoretical analysis presented in [3-9] is applied to the training and classification of artificial, a pseudo-random PRI radar sequence. The training of a HMM will first be studied when there are no errors in the sequences and its performance is tested as a function of the number of states in the model. The effects of both observation errors as well as loss of time synchronization on recognition performance will be investigated. Finally, the recognition performance of a HMM will be compared to that of a conventional cross-correlation technique. This paper is organized as follows. Section 2 introduces the basic concept of operation of a pseudo-random PRI radar. In Section 3, the performance of the HMM is evaluated as a function of the number of states when there are no errors in the received sequence. From the analysis, the best number of states used for training and recognition is obtained. In Section 4, the effects of both observation errors as well as loss of time synchronization on recognition performance are investigated. In Section 5, the recognition performance of a HMM is compared to that of a conventional cross-correlation technique. Section 6 will summarize the main conclusions.

\section{Pseudo-Random Sequences:}

Modern radar systems will change their transmitting signal's characteristic in several ways in order to maximize the performance. These can include changing the signal's carrier frequency, using different pulse modulation techniques, and changing the PRI of the signals [10]. Since we are only using PRI information for our modeling purposes, this last characteristic is obviously of great importance. Figure 3 shows an observed sequence from a pseudo-random PRI radar.

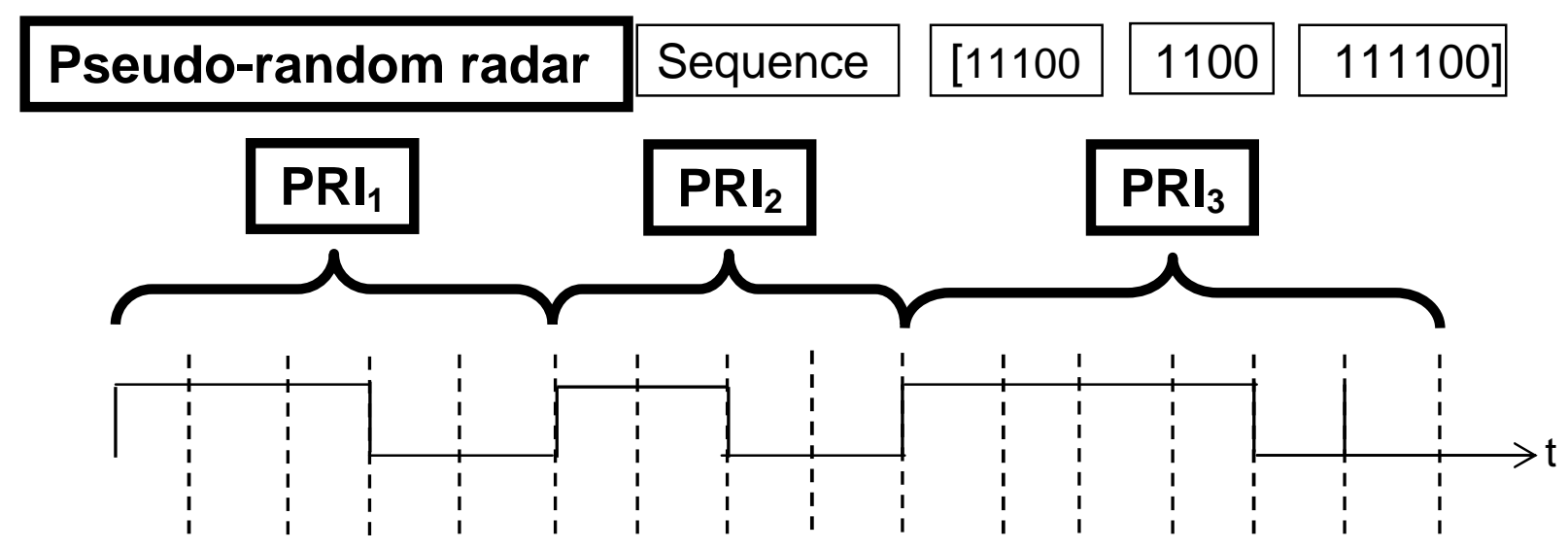

Figure (3): Observed sequence from a pseudo-random PRI radar. 
Using different PRIs is an advantage for reducing the effects of blind speed in Moving Target Indicator (MTI) radar systems, resolving range ambiguities in Pulse Doppler Radars, when switching between search and tracking modes. Because, we do not know how often an active enemy radar is going to switch between different PRIs or under what circumstances it would do so, an incoming signal will appear to consist of a random concatenation of different periodic pulse trains. This is the theory behind the way in which artificial pseudo random radar signals are programmed and used to investigate HMM recognition performance for this paper. A total of eight radars are created, each with the ability to produce a given number of different pulse trains with a corresponding pre-determined probability of occurrence. For example, one of the radars is programmed to be able to generate the following two pulse trains which we call words: word 1: 01 with probability $1 / 2$, word 2: 001 with probability $1 / 2$ (Appendix A).

\section{Number of States}

As in the deterministic case [2], the choice of the number of states to use for a HMM trained on a pseudo-random sequence is critical to its recognition performance. Unfortunately, this choice is not as easy for pseudo-random sequences because a perfect model is much harder to find. Since the training and observation sequences are not periodic, it is not easily apparent how many states are needed for a perfect training probability. Even with training probability equal to 1, however, there is no guarantee of perfect recognition for uncorrupted observation sequences as was the case for deterministic signals [2], since the observation sequences will not necessarily be the same as the training sequence. Furthermore, results vary depending on the length of the training sequence. A HMM trained on a short sequence will not perform as well as one trained on a long one because it does not have enough information about the source. Where as deterministic sequence training only required two periods of the sequence to capture all the information, pseudo-random signals need to be much longer in order to reflect the true statistics of the radar. Long sequences and large number of states take an extraordinary amount of computing power for HMM training which means that investigating recognition performance as a function of states is a very slow process. To compound the problem of the extraordinary amount of computational time, MATLAB began having problems with number overflow when the number of states reaches approximately 100 .

Figure 4 shows how three sequences from the same sources can have very different training probabilities for a given number of states. The radar used is the same one introduced in Section 2 and the training sequences contain five words. 


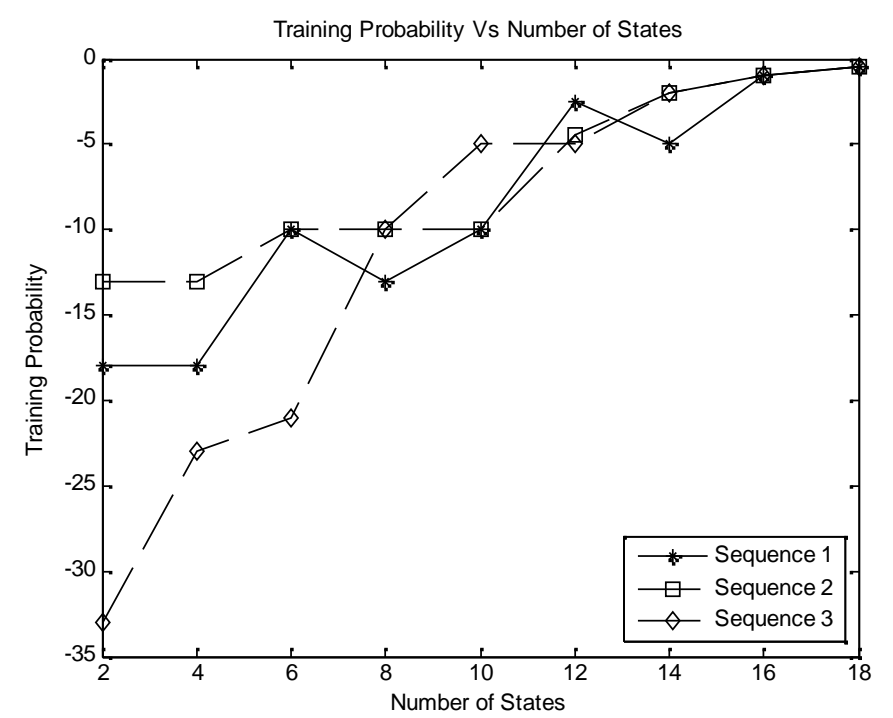

Figure (4): Training probability Vs number of states for pseudo random radar.

The large discrepancies in the training probability of each sequence underscore the fact that optimal parameters for pseudo-random sequence are not an easy task. Figure 5 shows the log recognition probability of the same three models trained using the previous three sequences as a function of the number of states for long (100 word) observation sequences generated from the same source.

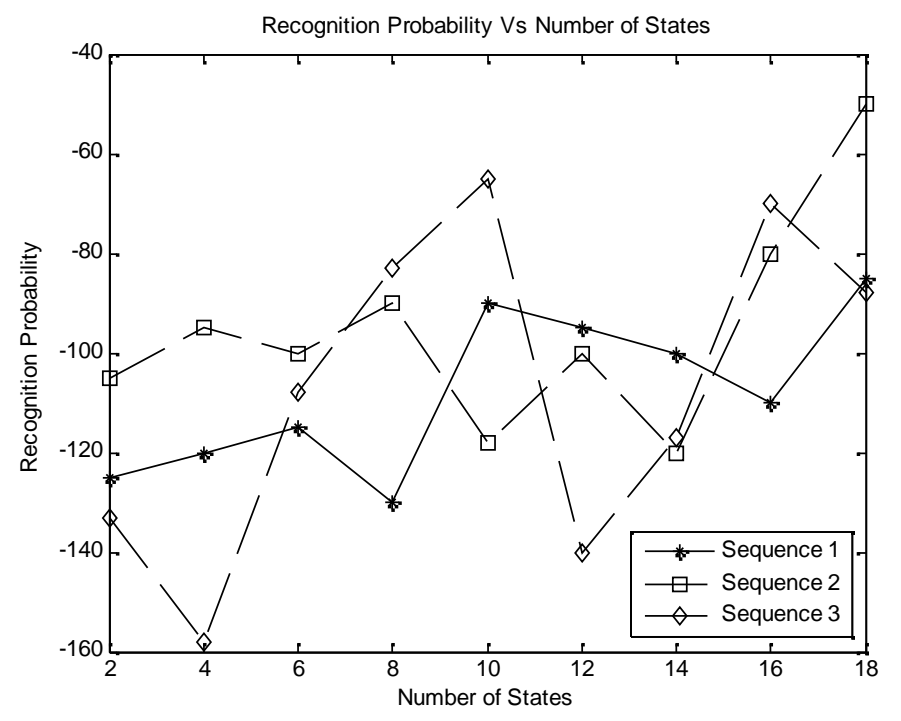

Figure (5): Recognition probability Vs number of states for pulsed radar

Contrary to intuition, the recognition probabilities degrade dramatically as the number of states increases. This is surprising since, it would seem, more states would allow the HMM to contain more information about the source resulting in better recognition performance. The fact that these models were only trained one five word sequences, however, means that $A$ and $B$ matrices do not contain state transition and output symbol probabilities that accurately describe this pseudo-random source. Much longer training sequences are, therefore, required to study this problem further. 
In a deterministic sequence, first order Markov process is used to model the stable PRI radar as presented in [3]. In pseudo-random radar we can not use this model because the radar consists of more than one word and there is more than one transition between words. Thus, we use an ergodic HMM to model the pseudorandom radar.

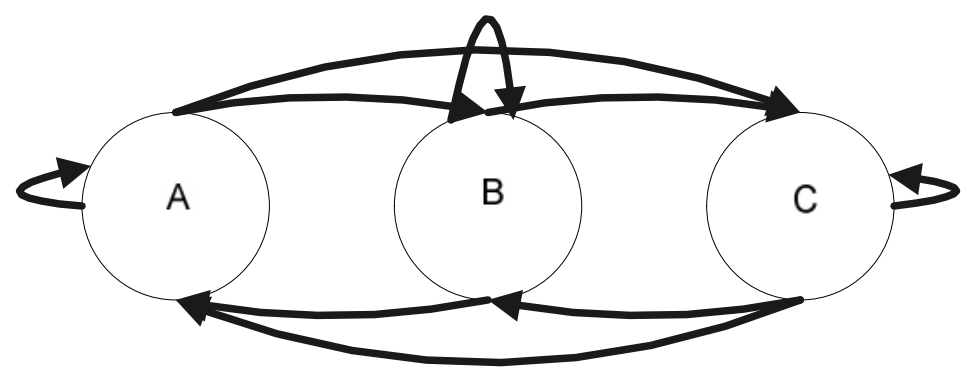

Figure (6): 3-State Ergodic HMM

As an example, the following artificial radar that contains three equiprobable words:

Word $1:[01]$ with probability $1 / 3$

Word $2:\left[\begin{array}{lll}0 & 0 & 1\end{array}\right]$ with probability $1 / 3$

Word $3:\left[\begin{array}{lll}1 & 0 & 1\end{array}\right]$ with probability $1 / 3$

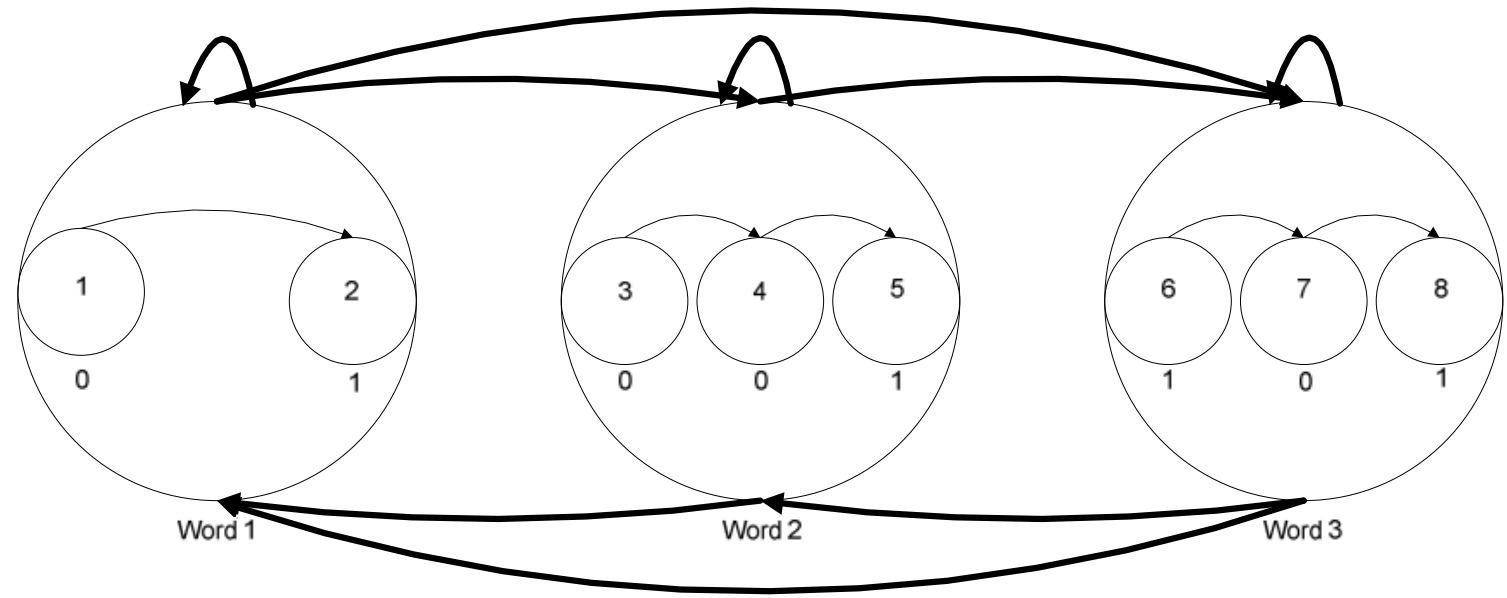

Figure (7): Ergodic HMM for pseudo-random PRI radar

The corresponding HMM has the following parameters

$$
\begin{aligned}
A & =\left[\begin{array}{cccccccc}
0 & 1 & 0 & 0 & 0 & 0 & 0 & 0 \\
1 / 3 & 0 & 1 / 3 & 0 & 0 & 1 / 3 & 0 & 0 \\
0 & 0 & 0 & 1 & 0 & 0 & 0 & 0 \\
0 & 0 & 0 & 0 & 1 & 0 & 0 & 0 \\
1 / 3 & 0 & 1 / 3 & 0 & 0 & 1 / 3 & 0 & 0 \\
0 & 0 & 0 & 0 & 0 & 0 & 1 & 0 \\
0 & 0 & 0 & 0 & 0 & 0 & 0 & 1 \\
1 / 3 & 0 & 1 / 3 & 0 & 0 & 1 / 3 & 0 & 0
\end{array}\right], \\
B & =\left[\begin{array}{cccccccc}
1 & 0 & 1 & 1 & 0 & 0 & 1 & 0 \\
0 & 1 & 0 & 0 & 1 & 1 & 0 & 1
\end{array}\right],
\end{aligned}
$$




$$
\pi=\left[\begin{array}{llllllll}
1 / 3 & 0 & 1 / 3 & 0 & 0 & 1 / 3 & 0 & 0
\end{array}\right] .
$$

It is worth noting that the training probability is computed using the forwardbackward procedure as follows:

$$
\begin{gathered}
\alpha_{1}(i)=\pi_{i} b_{i}\left(o_{1}\right) ; 1 \leq i \leq N \\
\alpha_{t+1}(j)=\left[\sum_{i=1}^{N} \alpha_{t}(i) a_{i j}\right] b_{j}\left(o_{t+1}\right) ; 1 \leq t \leq T-1,1 \leq j \leq N
\end{gathered}
$$

where $\boldsymbol{N}$ is the number of states in the HMM, and $\boldsymbol{T}$ is the observed sequence length. Finally the sum of the terminal forward variables over all states in the model defined as the probability of producing the observed sequence $\boldsymbol{O}$ given the HMM model $\lambda$.

$$
p\left(O \mid \lambda^{(l)}\right)=\sum_{i=1}^{N} \alpha_{T}(i)
$$

Figure 8 shows the $\log$ recognition probability of two HMMs. The first one represents the model of the source of the observation sequence (Radar 1), and the other one is the competing model (Radar 3). Both HMMs are trained using a sequence of 100 word length.

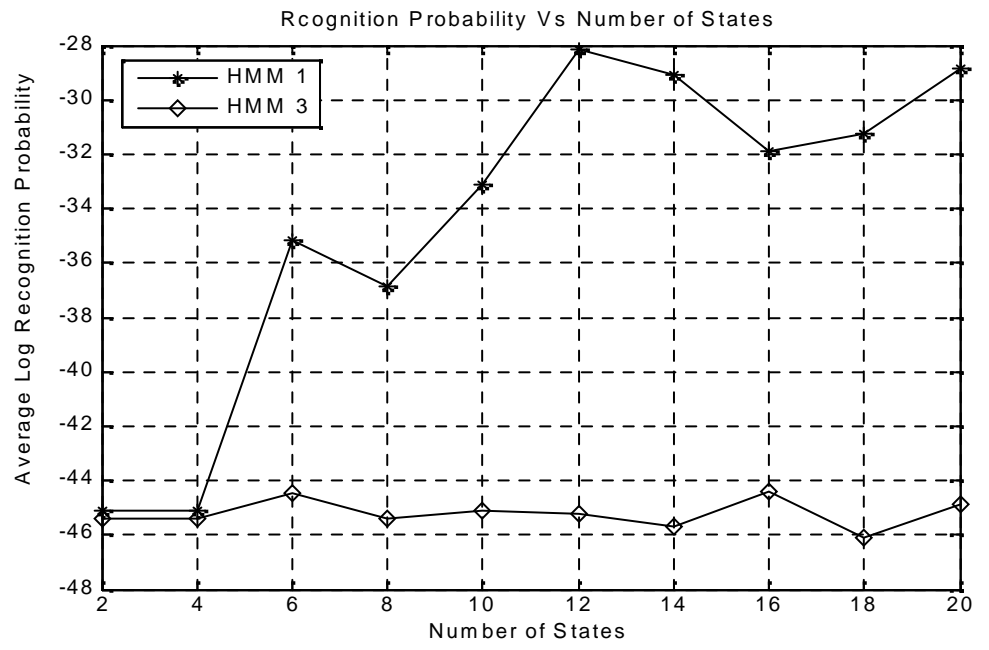

Figure (8): Recognition probabilities of correct $\left(H M M_{1}\right)$ and competing $\left(\mathrm{HMM}_{3}\right)$ model

It is clear that better recognition performance occurs when the difference between a correct and competing model's recognition probability is too large. Therefore, the number of states in the model should be chosen such that this difference will be maximized. Figure 9 shows this difference for the two models used in Figure 8. 


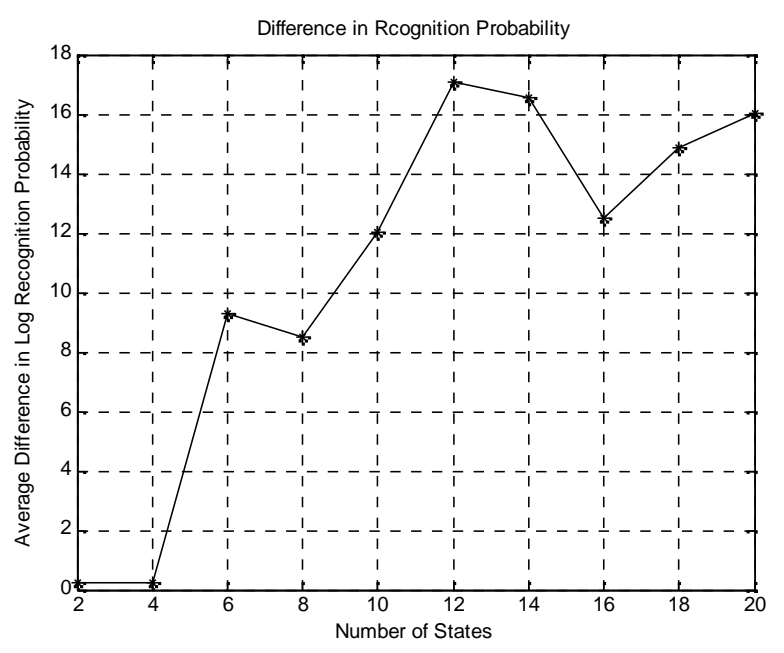

Figure (9): Difference in recognition probabilities for $\mathrm{HMM}_{1}$ and $\mathrm{HMM}_{3}$

This plot suggests that beyond a certain number of states (12 in this case), the recognition performance will not be improved. If this is the case taken, then knowing the threshold number of states becomes critical. Choosing a number that is too small will result in a poor performance while too large number will entail extremely long training times. Although this latter concern is not as important since processing power will be inevitably improved, it would still be advantageous to be able find some method for determining the minimum required number of states. Figure 10 plots the recognition curves for two other pseudo-random radars while Figure 11 shows the difference in the recognition probabilities.

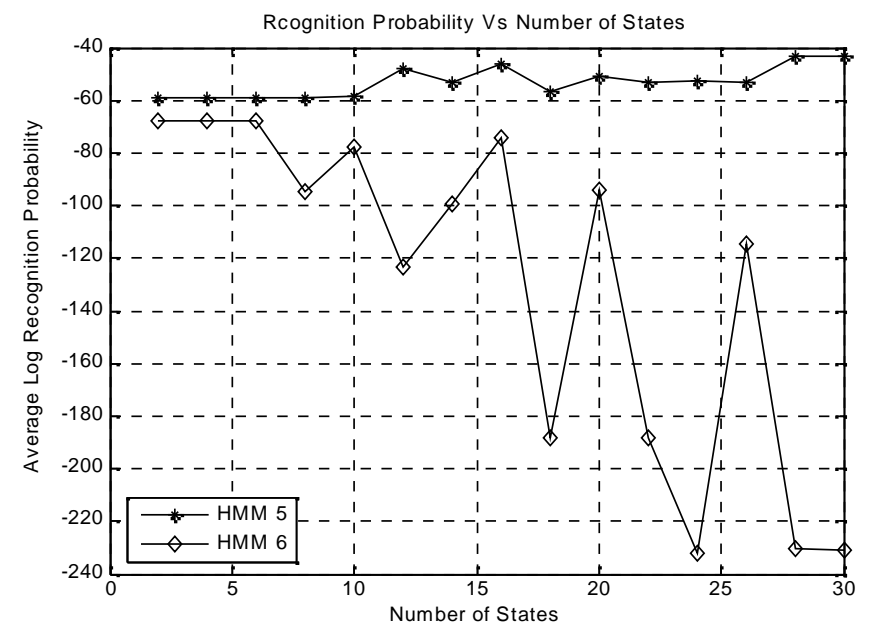

Figure (10): Recognition probabilities of correct $\left(H M_{5}\right)$ and competing $\left(H M M_{6}\right)$ model 


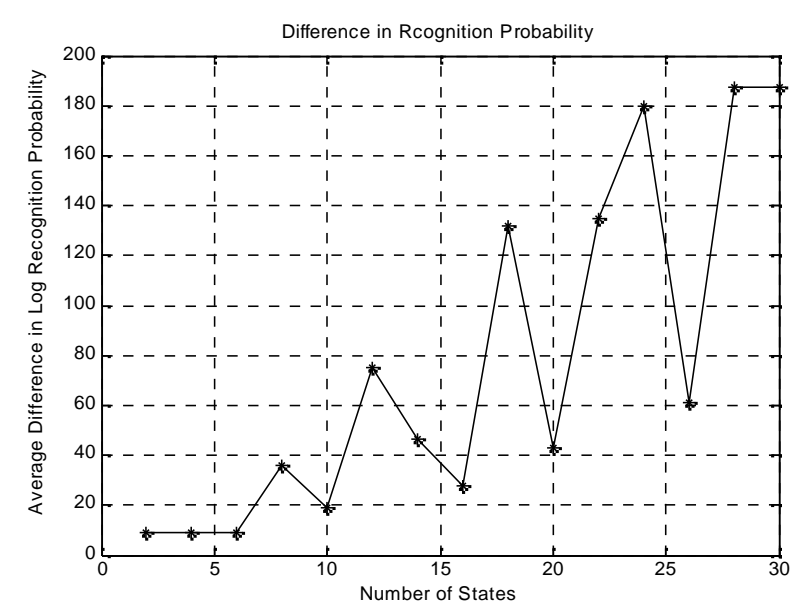

Figure (11): Difference in recognition probabilities for $\mathrm{HMM}_{5}$ and $\mathrm{HMM}_{6}$

Once again it appears that there is a threshold number of states beyond which the recognition performance of a HMM seems to plateau (28 state in this case). The correct models in Figure 7 and 9 were from radars 1 and 5. Table 1 outlines their PRI information

Table 1: Radar 1 and 5 PRI information

\begin{tabular}{|c|c|c|c|}
\hline \multicolumn{2}{|c|}{ Radar 1 } & \multicolumn{2}{|c|}{ Radar 5 } \\
\hline \hline Word & Probability & Word & Probability \\
\hline \hline 01 & 0.25 & 0000101 & 0.25 \\
\hline 001 & 0.25 & 0101 & 0.25 \\
\hline 011 & 0.25 & 110110101 & 0.25 \\
\hline 1001 & 0.25 & 00011001 & 0.25 \\
\hline
\end{tabular}

If we look at the total word length for each radar we see that for radar 1 it is equal to $(2+3+3+4)=12$ and for radar 5 we $\operatorname{get}(7+4+9+8)=28$. It is interesting that these two total lengths correspond exactly to the number of states where the recognition performance curves level reaches their peak values as shown in Figure 7 and Figure 9. This is similar to the deterministic case [2] where the best number of states was also equal to the word length (which was the period of the sequence).

A preliminary conclusion would be to say that approximate expression for the minimum required number of states is simply the value of the total signal's word length which is similar to PRI frame of the staggered (Pseudo-Random) PRI radar.

$$
N_{\text {min }}=\sum_{i=1}^{M} T_{i}
$$

where $\mathrm{M}$ is the total number of words and $\mathrm{T}_{\mathrm{i}}$ is the number of symbols in the word.

A closer look at Table 1, however, shows that both radars have words that are subset 
of other words. Radar 3, for example, can produce 01 , which can be found in words 1,2 and 3 as well as 4 if it repeats itself. This may have an effect on what the minimum number of states should be. To test this, it was decided to compute the recognition performance of a correct and competing model trained on pseudoorthogonal words (Appendix B). That is, the '1's and '0's in each word are chosen at random so that it would be extremely improbable for one word to be a subset of any other. To this end, two pseudo-random orthogonal radars are created both with an equiprobable five-word code book with each word being 10, 12, $14,16,18$ symbols in length. Figure 12 shows the recognition probability for the two HMMs, Figure 13 plots the difference in these probabilities.

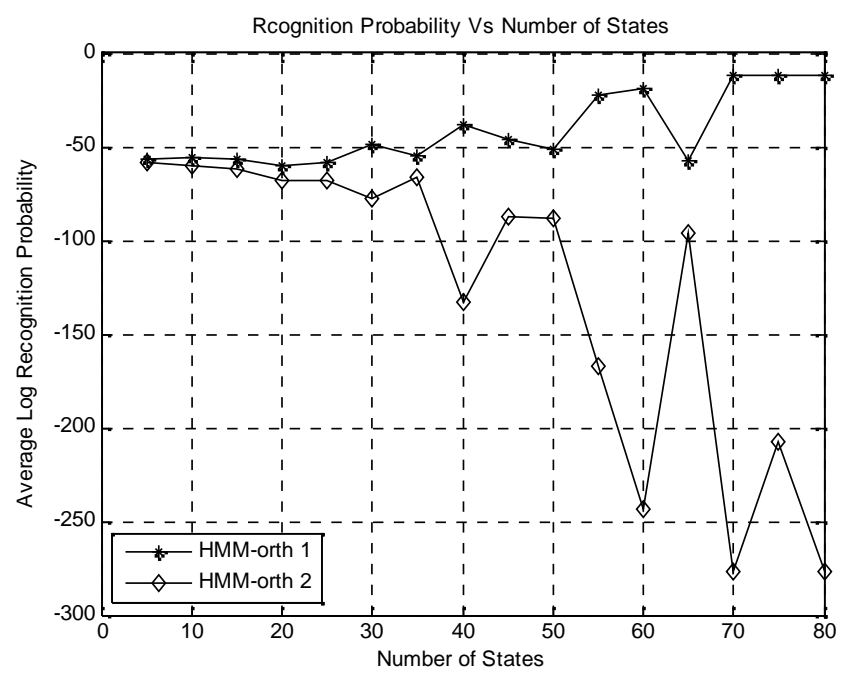

Figure (12): Recognition probabilities of correct and competing models with pseudo orthogonal sequences

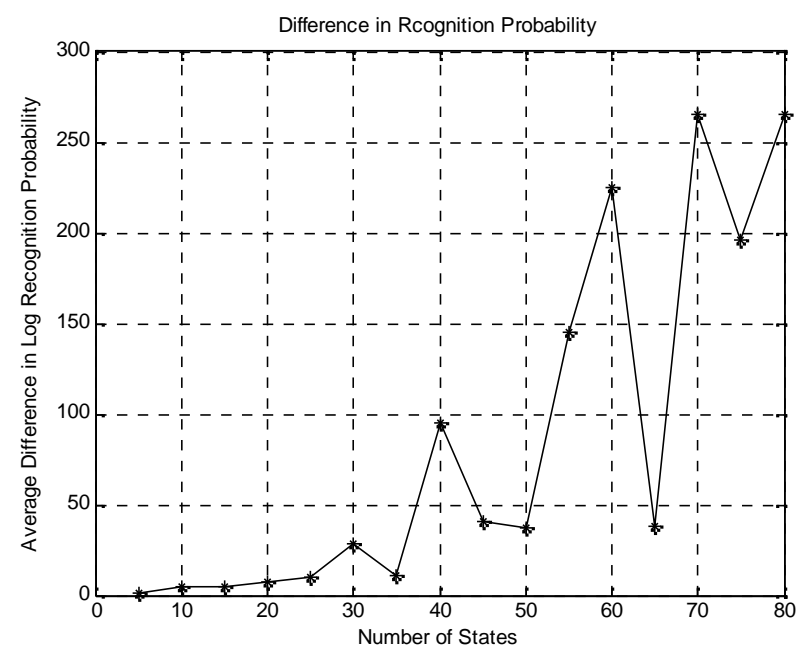

Figure (13): Difference in Recognition Probabilities

Figure 13 seems to indicate that more states provide better recognition probability. Long time is required to run the simulation. It took approximately 25 hours to be completed. The following two factors account for this long processing time: 
1- For each state, several HMMs must be computed since the Baum-Welsh method only guarantees a local maximum training probability that is determined by the initial conditions on $A, B$ and $\pi$. This simulation took the best one of 25 simulations.

2- The recognition probabilities per state for both models must be computed for many observation sequences and then averaged in order to get an accurate plot. 500 independent test sequences were generated per state.

A second simulation was run in which both the training and the recognition probabilities of two HMMs were recorded as a function of the number of states. In this simulation both HMMs modeled pseudo-random sources consisting of five equiprobable, randomly chosen words of length 10, 12, 14, 16 and 18 symbols each. In addition, the simulation went up to 100 states to see if a plateau occurs in the recognition probability beyond 70 . Figure 14 shows the training probabilities of both HMMs, Figure 15 shows the actual recognition probabilities of the correct and competing models, and Figure 16 shows the difference in recognition probabilities.

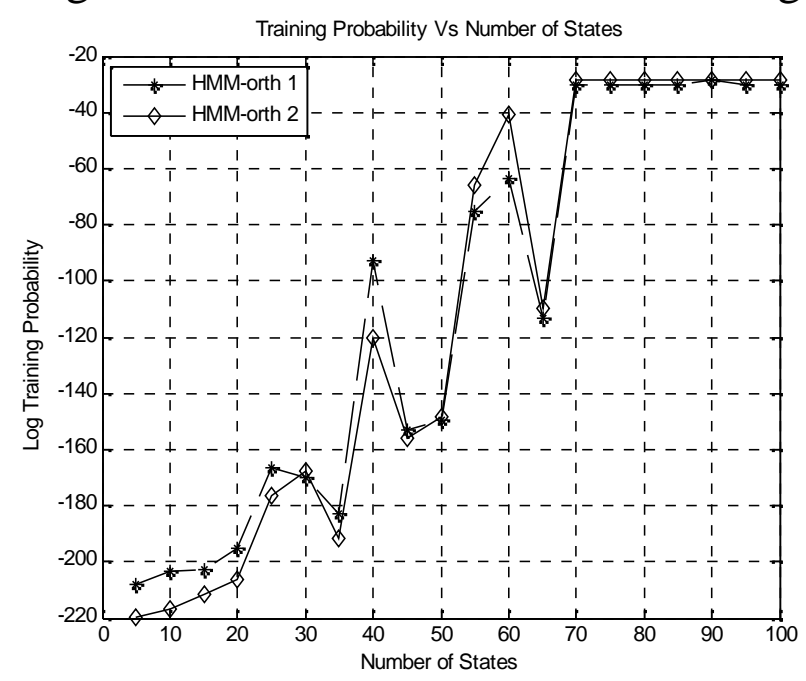

Figure (14): Training probability Vs number of states for pseudo-orthogonal sequences

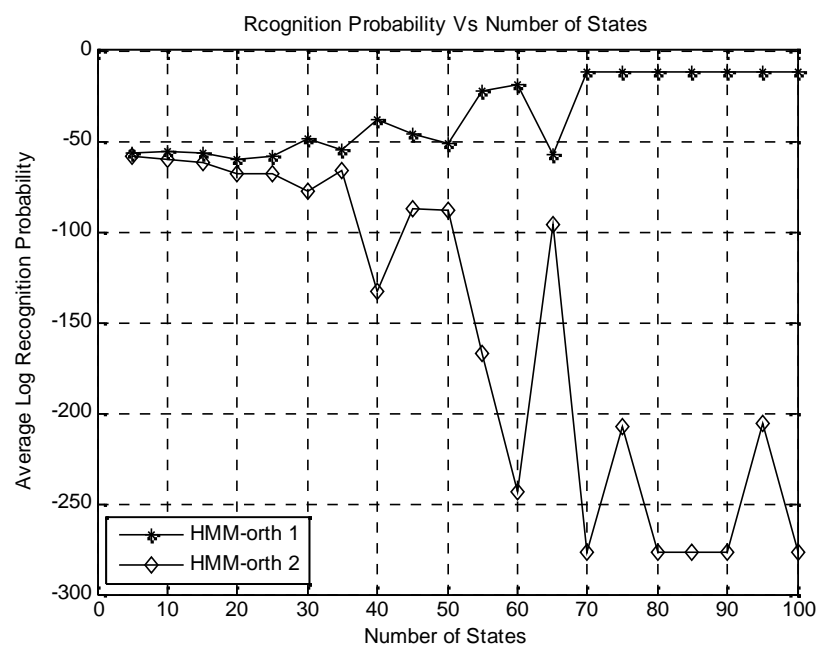

Figure (15): Recognition Probability Vs Number of 
States for Pseudo-Orthogonal Sequences

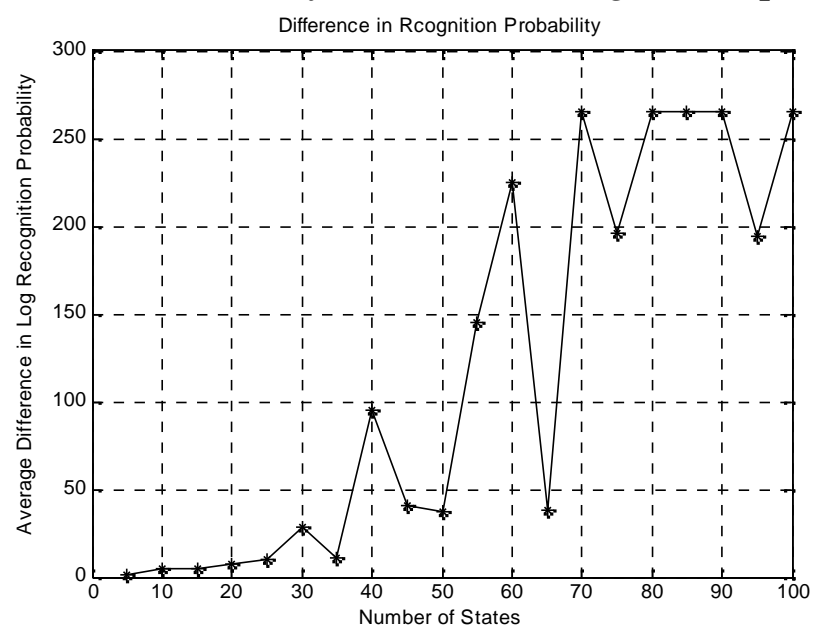

Figure (16): Difference recognition probability Vs number of states for pseudo-orthogonal sequences

\section{Recognition Performance with Sequence Errors and with Loss of Synchronization:}

In practice, the first received symbol will not necessarily be the beginning symbol of a word transmitted by a radar. In fact, more often than not, the receiver will encounter a signal in mid-sentence so it is important to understand how this will affect the recognition performance of a HMM. Figure 17 shows the false recognition rates of two observation sequences originating from radar 3 and 7 . In both cases, the beginning of the sequence was truncated by 5 symbols.
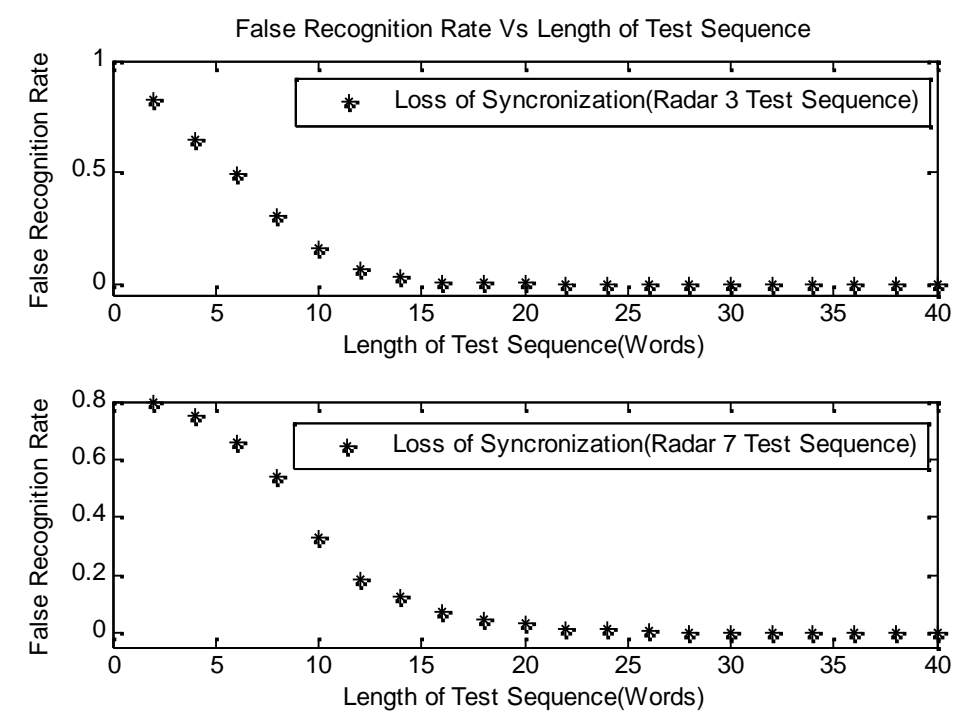

Figure (17): False recognition rate with loss of synchronization

Figure 18 shows the false recognition rates for the same two radars, but with no loss of synchronization for comparison purposes. It is clear that synchronization loss will 
increase the probability of incorrectly classifying an observation sequence when the observation sequence is short.
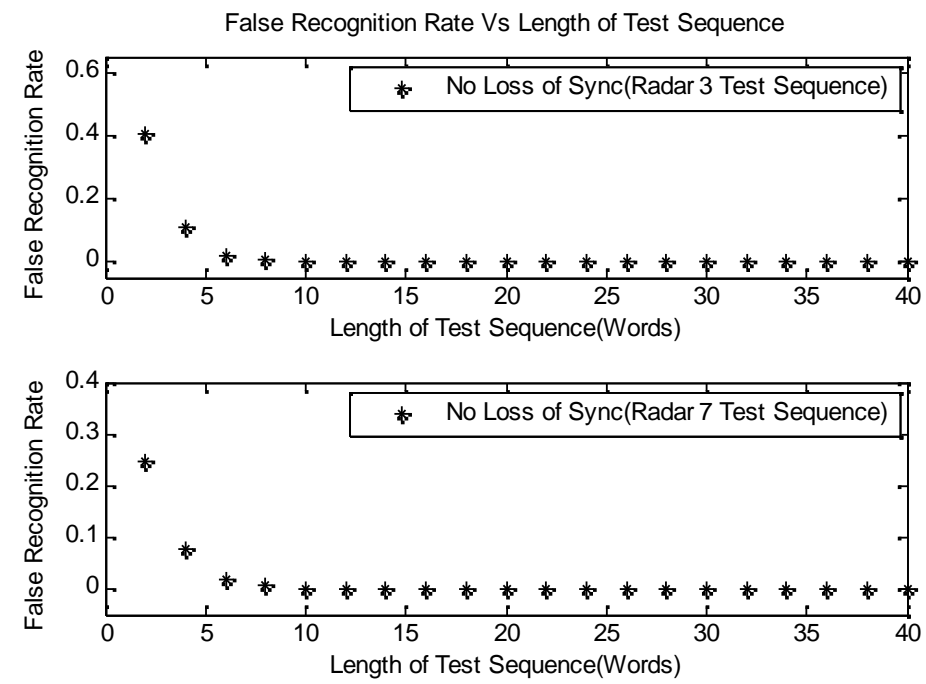

Figure (18): False recognition rate without loss of synchronization

\section{Comparison to Cross-Correlation:}

The standard method for estimating the degree to which two sequences are correlated is cross-correlation. The cross-correlation between two real, continuous functions $f(t)$ and $g(t)$ is defined as

$$
f(t) * g(t)=\int_{-\infty}^{\infty} f(t) g(t-\tau) d \tau
$$

The maximum value of equation 6 denotes the time delay $\tau$, where the two functions are most closely correlated (line up the best). The cross-correlation between two discrete pulse trains $f(k)$ and $g(k)$ simply replaces the integral by a discrete summation.

Because cross-correlation has traditionally been used in pattern recognition applications, the question as to what improvement a HMM can provide is of a fundamental importance. If a HMM offers no improvement over cross-correlation then it would not make sense to use this stochastic modeling approach due to the greatly increased computation complexity. Figure 19 shows the false recognition rates using HMMs (forward-backward algorithm) and cross-correlation when radar 6 is the observation sequence generator. In this simulation there is no observation error or retraining. 


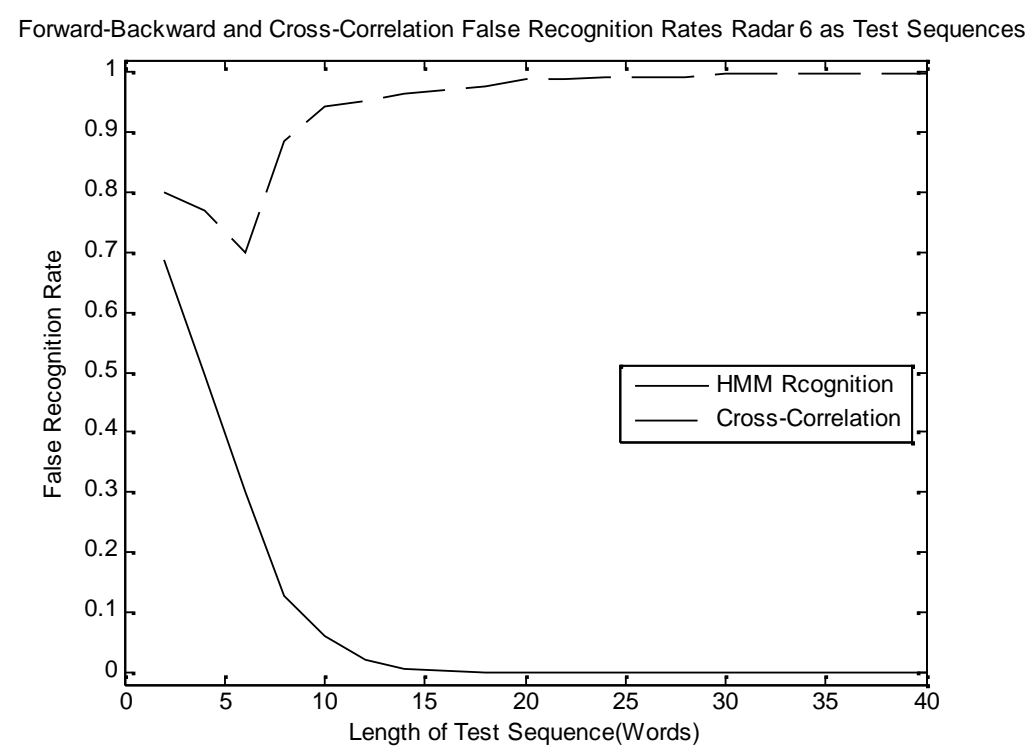

Figure (19): HMM and cross-correlation false recognition rates comparison (radar 6 as test sequence)

Figure 20 shows the forward-backward and cross-correlation false recognition rates with radar 4 as a test sequence. In this simulation there are observation errors (10\% dropped $5 \%$ spurious pulses) and retraining.

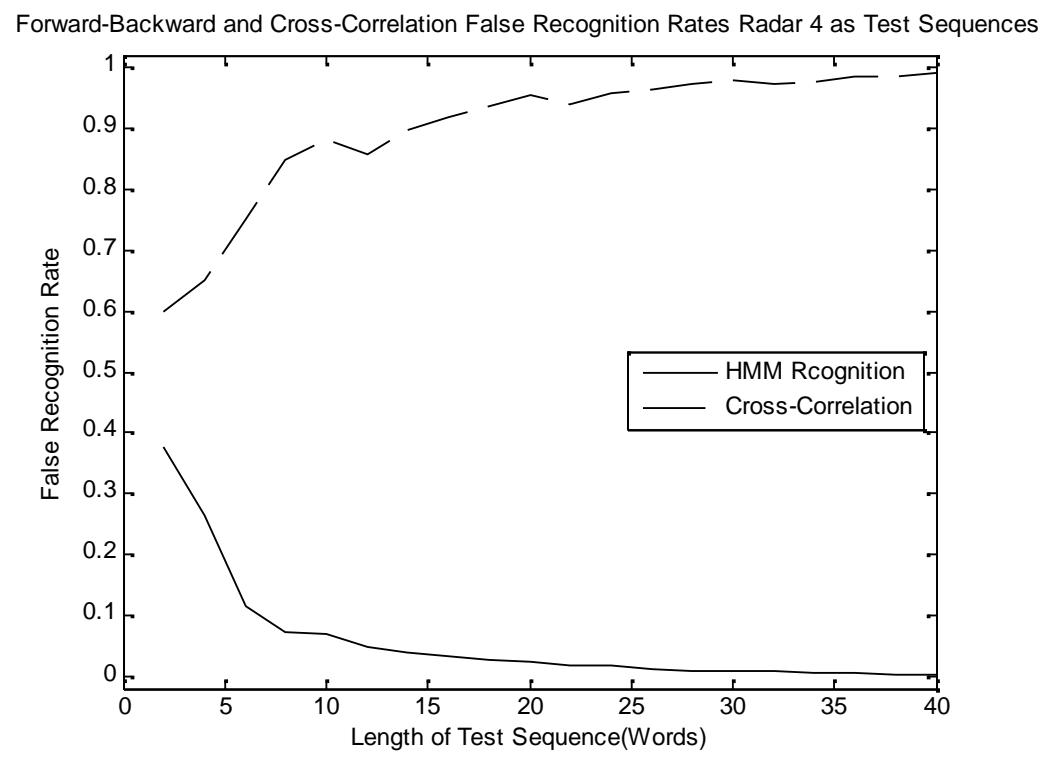

Figure (20): HMM and Cross-Correlation False Recognition Rates Comparison (Radar 4 as Test sequences)

It is clear from the last two figures that the recognition performance for HMMs is far superior to that of simple signal cross-correlation as expected.

\section{Conclusions:}

This paper, switches the focus of HMM training and recognition to pseudo-random sequences. It is shown that the recognition performance for HMMs trained on 
pseudo-random sequences is, in general, quite acceptable provided that the training and the observation sequences are sufficiently long. Both the training and the recognition performance of a HMM are investigated as a function of the number of states, $N$, included in the model. Unlike in the deterministic case, there does not appear to be a simple methodology for choosing the best value of $\mathrm{N}$ for a pseudorandom sequence. It is shown that, the idea of choosing the number of states in the model, $N$, according to the radar words length is promising. It is highly probable that good values of $N$ will be dependent on both the nature of the training sequence and the other competing HMMs within a given threat library. Some HMMs may be very different from each other and this means that the value of $N$ will not have too great effect on the recognition performance. On the other hand, as was seen from the used artificial pseudo-random sequences, some HMMs may turn out to be very close so that the number of states becomes a critical parameter especially for the recognition of short, error-corrupted sequences. Efforts to find how many states should be included in a given HMM were hampered, however, by the long computing times needed for HMM training. When compared to cross-correlation, it was found that HMMs are far superior in recognition even if the test sequences contain no errors. This can be attributed to the rich mathematical structure that HMMs possess and to their ability to model pseudo-random sequences.

\section{References:}

[1] Hossam E. Abou-Bakr Hassan, Khairy Elbarbay, Mohamed Asaad AbdElrazek "Identification of Pulsed Radar using Hidden Markov Model", The $12^{\text {th }}$ International Conference on Aerospace Sciences and Aviation Technology, Military Technical College May, Cairo, Egypt, 2007.

[2] Hossam E. Abou-Bakr Hassan, Ahmad El-Mahdy, Mohamed Asaad AbdElrazek "Performance Evaluation of Pulsed Radar Identification using Hidden Markov Model", The $12^{\text {th }}$ International Conference on Aerospace Sciences and Aviation Technology, Military Technical College May, Cairo, Egypt, 2007.

[3] Rabiner, L.R., "A tutorial on hidden Markov models and selected applications in speech recognition", Proc. IEEE, Vol. 77, No. 2, pp. 257285, February, 1989.

[4] Roe, D.B., Wilpom, J.G., "Whither speech recognition: The next 25 years", IEEE Comm. Magazine, pp. 54-62, November, 1993.

[5] Picone, J.W., "Signal modeling techniques in speech recognition', Proc. IEEE, Vol. 81, No. 9, p. 1216, September, 1993.

[6] Junkowitsch, J., Neubauer, L., Kuske, G., "A new keyword spotting algorithm with pre-calculated optimal thresholds", Proc. $4^{\text {th }}$ Int. Con. on Spoken Language Processing, Philadelphia, PA., 3-6, October, 1996.

[6] Matsui, T., Nishitani, T., Firui, S., "Robust methods of updating model and a priori threshold on speaker verification", Proc. $21^{\text {st }}$ IEEE Int. Con. on Acoustics, Speech, and Signal Processing, Atlanta, GA., 7-10, May, 1996. 
[7] Genoval, D., Moreira, M., Mayoraz, E., "Test text dependent speaker verification using binary classifiers", 1998 IEEE International Conference on Acoustics Speech, and Signal Processing, May, 1998.

[8] Juang, B.-H. and Rabiner, L., "A probabilistic measure for hidden Markov models", AT\&T Technical Journal., Vol. 64, No. 2, pp. 391-409, February 1985.

[9] Jen-Tzung Chien, Senior Member, Sadaoki Furui "Predictive Hidden Markov Model Selection for Speech Recognition" Proc. IEEE, 2005.

[10] Merril Skolnik, "Radar Handbook Second Edition", McGraw-Hill, Inc., 1990.

APPENDIX A :

Table 2: Pseudo-Random radars 1 through 8

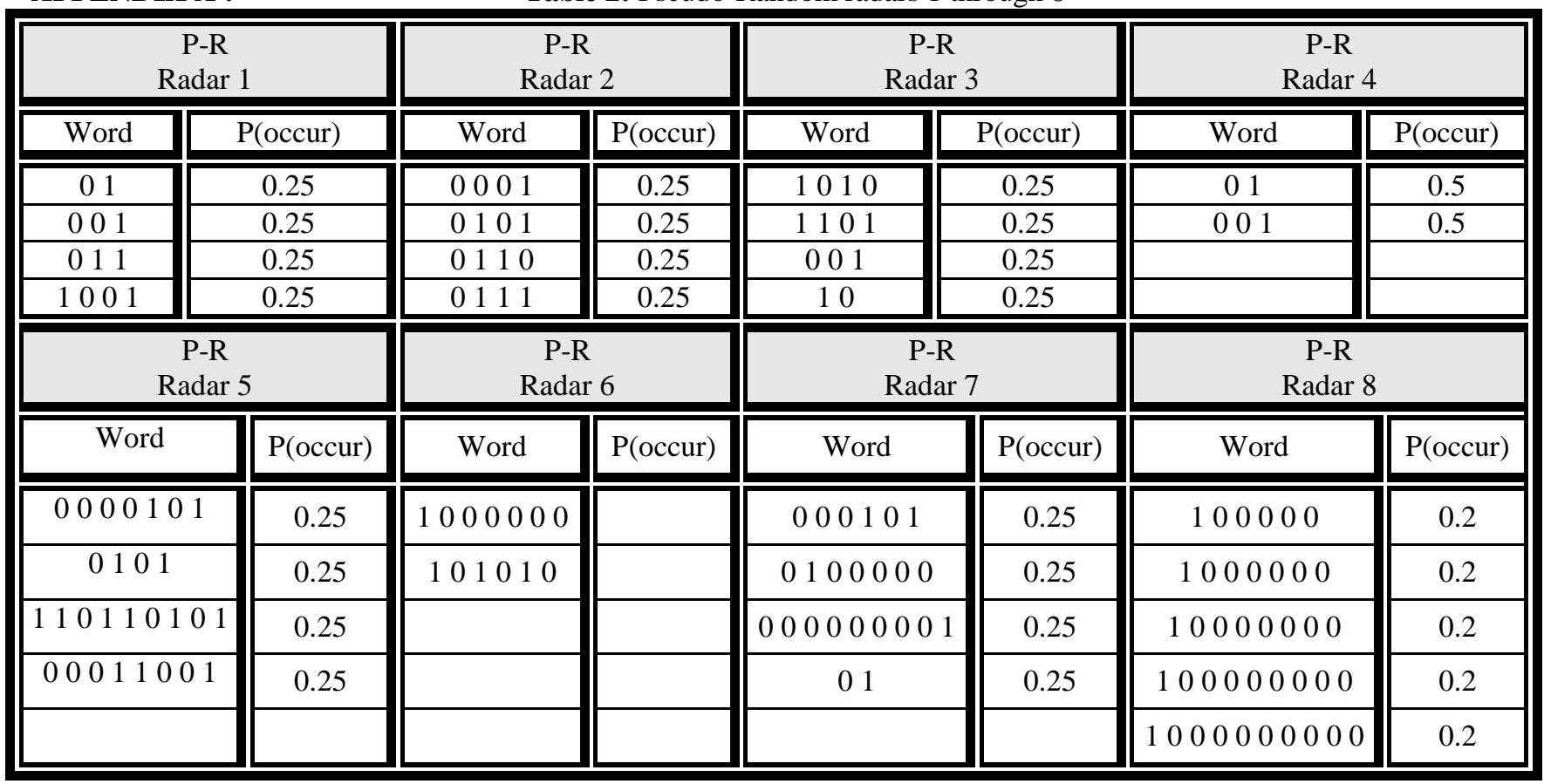

APPENDIX B:

Table 3 :Pseudo-Orthogonal Radars

\begin{tabular}{|c|c|c|c|c|c|c|c|c|c|c|c|c|c|c|c|c|c|c|}
\hline \multicolumn{18}{|c|}{$\begin{array}{c}\text { Radar } 9 \\
\text { Word }\end{array}$} & \multirow{2}{*}{$\begin{array}{c}\mathbf{P}(\text { occur }) \\
0.2 \\
\end{array}$} \\
\hline 1 & 1 & 0 & 0 & 0 & 0 & 0 & 0 & 1 & 0 & & & & & & & & & \\
\hline 0 & 0 & 0 & 1 & 0 & 1 & 0 & 0 & 1 & 0 & 1 & 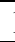 & & & & & & & 0.2 \\
\hline 0 & 1 & 1 & 1 & 0 & 0 & 1 & 0 & 0 & 0 & 0 & 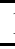 & 1 & 0 & & & & & 0.2 \\
\hline 1 & 0 & 1 & 1 & 1 & 1 & 1 & 1 & 1 & 1 & 1 & 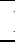 & 0 & 0 & 1 & 0 & & & 0.2 \\
\hline 0 & 1 & 1 & 0 & 1 & 0 & 1 & 0 & 0 & 0 & 1 & & 1 & 1 & 1 & 0 & 0 & 1 & 0.2 \\
\hline \multicolumn{18}{|c|}{$\begin{array}{c}\text { Radar } 10 \\
\text { Word } \\
\end{array}$} & $\mathbf{P}($ occur $)$ \\
\hline 1 & 1 & 1 & 1 & 1 & 1 & 0 & 1 & 0 & 0 & & & & & & & & & 0.2 \\
\hline 0 & 0 & 0 & 0 & 1 & 0 & 0 & 1 & 0 & 1 & 1 & & & & & & & & 0.2 \\
\hline 0 & 0 & 0 & 0 & 1 & 1 & 0 & 1 & 0 & 0 & 0 & & 0 & 1 & & & & & 0.2 \\
\hline 0 & 0 & 1 & 1 & 0 & 1 & 1 & 0 & 1 & 1 & 1 & & 0 & 1 & 1 & 0 & & & 0.2 \\
\hline 1 & 1 & 0 & 1 & 1 & 1 & 0 & 1 & 1 & 0 & 1 & ( & 0 & 0 & 0 & 0 & 1 & 1 & 0.2 \\
\hline
\end{tabular}

